\title{
Underlying mechanism of numerical instability in large eddy simulation of turbulent flows
}

\author{
Masato Idd* \\ Collaborative Research Center of Frontier Simulation Software for Industrial Science, \\ Institute of Industrial Science, University of Tokyo, \\ 4-6-1 Komaba, Meguro-Ku, Tokyo 153-8505, Japan \\ Nobuyuki Taniguchi \\ Information Technology Center, University of Tokyo, \\ 2-11-16 Yayoi, Bunkyo-Ku, Tokyo 113-8658, Japan
}

\begin{abstract}
This paper extends our recent theoretical work concerning the feasibility of stable and accurate computation of turbulence using a large eddy simulation [Ida and Taniguchi, Phys. Rev. E 68, 036705 (2003)]. In our previous paper, it was shown, based on a simple assumption regarding the instantaneous streamwise velocity, that the application of the Gaussian filter to the incompressible Navier-Stokes equations can result in the appearance of a numerically unstable term that can be decomposed into positive and negative viscosities. That result raises the question as to whether an accurate solution can be achieved by a numerically stable subgrid-scale model. In the present paper, based on assumptions regarding the statistically averaged velocity, we present similar theoretical investigations to show that in several situations, the shears appearing in the statistically averaged velocity field numerically destabilize the fluctuation components because of the derivation of a numerically unstable term that represents negative diffusion in a fixed direction. This finding can explain the problematic numerical instability that has been encountered in large eddy simulations of wall-bounded flows. The present result suggests that this numerical problem is universal in large eddy simulations, and that if there is no failure in modeling, the resulting subgrid-scale model can still have unstable characteristics; that is, the known instability problems of several existing subgridscale models are not something that one may remove simply by an artificial technique, but must be taken seriously so as to treat them accurately.
\end{abstract}

PACS numbers: 47.11.+j, 47.27.Eq, 47.10.+g, 83.85.Pt

\section{INTRODUCTION}

Turbulence is one of the unsolved problems of physics [1]. Because a complete theoretical description has not yet been achieved even for a relatively simple flow configuration, numerical simulations are commonly used as a powerful tool for analyzing turbulent flows. Typical numerical approaches include the direct numerical simulation (DNS), the Reynolds-averaged Navier-Stokes (RANS), and the large eddy simulation (LES). In DNS, all the scales of motion in the turbulent flows are resolved by sufficiently fine computational grids, whereas in RANS, only the evolution of mean quantities is solved. LES is an intermediate technique between these approaches, directly solving the large scales but modeling small-scale eddies by employing a subgrid-scale (SGS) model (or a subfilter-scale model) that approximately accounts for the effects of the small scales on the large scales [2]. Because LES enables us to solve time-dependent

\footnotetext{
*E-Mail: $\quad$ ida@icebeer.iis.u-tokyo.ac.jp URL http://ktolab.iis.u-tokyo.ac.jp/ida/index.htm Present address: Center for Promotion of Computational Science and Engineering, Japan Atomic Energy Research Institute, 6-93 Higashi-Ueno, Taito-ku, Tokyo 110-0015, Japan; E-Mail: ida@koma.jaeri.go.jp.
}

large-scale turbulent flow problems with a relatively small computational time and storage compared to those required for DNS, this technique has recently been used not only for academic studies but also for practical industrial flow computations that need time-dependent solutions.

One of the major problems of LES is numerical instability. As is already known, several existing SGS models (e.g., the tensor-diffusivity 3], the scale-similarity [4], and the dynamic Smagorinsky [5] models), which have been constructed based on a filtering procedure and the statistical properties of turbulence, have a numerically unstable property, and hence some artificial numerical treatments (e.g., smoothing or clipping of the SGS stress) have been incorporated so as to guarantee numerical stability $[2,5,6,6,8,8]$. While the mechanisms of these models' unstable behaviors have been described in the literature (e.g., Refs. 2, [8, 9, 10, 11]), the underlying reason as to why the SGS models have an unstable property has, to the authors' knowledge, not yet been fully clarified. To construct an excellent SGS model that is free from artificial, unphysical numerical treatments and has applicability to a wide range of flow configurations with high accuracy and robustness, it is necessary to elucidate the underlying mechanism of those unstable properties. Is this numerical problem caused by a failure in modeling or by other factors? To answer this question, it 
should be meaningful to consider a similar but idealized question whether a completely accurate SGS model, if it exists, would be numerically stable.

In Ref. [10], Leonard has shown that the tensordiffusivity model, which was derived by truncating an exact expansion series of the SGS stress terms and is thus exact under a certain condition, should behave unstably along the stretching directions of fluid motion. This unstable behavior results from the negative diffusivity of that model, which makes the governing equations ill-conditioned and leads to numerical instability when treated numerically by, e.g., finite difference methods. Winckelmans et al. [8] have performed several numerical experiments using the pure (and mixed) tensor-diffusivity model and have pointed out that the model behaves unstably under a wall-bounded flow condition, while it can provide a stable result for turbulent isotropic decay. In a recent paper [12] we have shown theoretically that the filtering procedure, which is the most fundamental component of LES, is a potential seed of the numerical instability in LES. Without having resorted to any SGS model, but based on a simple assumption regarding the streamwise component of flow velocity, we found that under a wall-bounded flow condition, the application of a Gaussian filter (one of the filters commonly used in LES [13]) to the Navier-Stokes equations results in the appearance of a numerically unstable term, a cross-derivative of the filtered velocity component, which can be decomposed into diffusion and negative-diffusion terms. The above findings indicate that apparently the application of Gaussian filtering stabilizes the physical properties of the governing equations, but this is not always the case. That result implies that even a completely accurate SGS model that perfectly reproduces the physical properties of the true SGS components might not always be numerically stable.

However, both Leonard's and Ida and Taniguchi's theoretical works are insufficient to fully clarify the mechanism of numerical instabilities in actual simulations. While those studies have only considered the instantaneous nature of the SGS model or of the SGS forces, it is possible that their time-averaged nature is dissipative and that stable simulations can thus be achieved. Indeed, as mentioned above, the tensor-diffusivity model, which has an unstable property, can provide stable solutions for an isotropic turbulent flow [8]. This observation suggests that further efforts must be made to improve our understanding of the numerical instabilities in LES.

In the present paper, we have extended our previous work so as to obtain more acceptable and reliable results and to show that this numerical problem is universal in LES based on Gaussian filtering. We have previously assumed that the instantaneous value of the streamwise velocity component is linearly proportional to the distance from a plane wall parallel to the bulk flow. In contrast, in the present paper our discussion assumes that the statistically averaged streamwise velocity component is linearly proportional to the distance from the wall, an assumption that is more realistic because this is the case of a viscous sublayer forming near a plane wall. Furthermore, we discuss cases where an axisymmetric swirl exists in the statistically averaged velocity field. As will be shown in the following section, a numerically unstable term with a time-independent coefficient can appear in both situations. This term is always unstable in a fixed range of directions, while the previously discussed unstable characteristics of the model and of the SGS terms depend on the directions of instantaneous stretching [10] or of instantaneous shears 12]. Therefore, the present theoretical result can more accurately explain the numerical instabilities frequently encountered in inhomogeneous flows that involve a strong steady shear in the mean velocity field.

This paper is organized as follows: In Sec. IB we reexamine the wall-bounded flow case, and in Sec. II C we extend our theory to swirling flow cases. In the appendixes, we provide additional notes on further extensions of our theory. Section

\section{THEORETICAL INVESTIGATIONS}

\section{A. Filtering approach}

Incompressible, viscous fluid flows are described by the Navier-Stokes equations, which read:

$$
\begin{gathered}
\frac{\partial u_{i}}{\partial t}+\frac{\partial u_{j} u_{i}}{\partial x_{j}}=-\frac{\partial p}{\partial x_{i}}+\nu \frac{\partial^{2} u_{i}}{\partial x_{j} \partial x_{j}} \quad \text { for } i=1,2,3 \\
\frac{\partial u_{i}}{\partial x_{i}}=0
\end{gathered}
$$

where Einstein's summation convention is assumed, and $u_{i}=u_{i}\left(x_{1}, x_{2}, x_{3}, t\right)$ are the velocity components, $p$ is the pressure divided by the constant fluid density, and $\nu$ is the kinematic viscosity. In LES, a filter is applied to this system of equations to separate the large and small scales. This filtering procedure is in general achieved by the following convolution:

$$
\bar{F}(x, \ldots, t)=\int_{X=-\infty}^{X=\infty} L(x-X) F(X, \ldots, t) d X,
$$

where $\overline{(\cdot)}$ denotes the filtered quantity, $x$ is an independent variable of an arbitrary function $F$, and $L(X)$ is the filter function. In the present study, we assume $L(X)$ to be the Gaussian function

$$
L(X)=\sqrt{\frac{\gamma}{\Delta^{2} \pi}} \exp \left(-\frac{\gamma X^{2}}{\Delta^{2}}\right),
$$

which satisfies $\int_{X=-\infty}^{X=\infty} L(X) d X=1$, where $\Delta$ is the filter width (assumed to be constant), and $\gamma$ is a real, positive constant. In the previous paper we set to $\gamma=1 / 2$ as 
done by Klimas for the Vlasov equation, while $\gamma=6$ is generally used in LES [13]; we adopt the latter value in the present study. Appling the filtering operation to Eqs. (1) and (2) yields

$$
\frac{\partial \bar{u}_{i}}{\partial t}+\frac{\partial \overline{u_{j} u_{i}}}{\partial x_{j}}=-\frac{\partial \bar{p}}{\partial x_{i}}+\nu \frac{\partial^{2} \bar{u}_{i}}{\partial x_{j} \partial x_{j}}
$$

or

$$
\begin{gathered}
\frac{\partial \bar{u}_{i}}{\partial t}+\frac{\partial \bar{u}_{j} \bar{u}_{i}}{\partial x_{j}}=-\frac{\partial \bar{p}}{\partial x_{i}}+\nu \frac{\partial^{2} \bar{u}_{i}}{\partial x_{j} \partial x_{j}}-\frac{\partial \tau_{i j}}{\partial x_{j}} \\
\tau_{i j} \equiv \overline{u_{i} u_{j}}-\bar{u}_{i} \bar{u}_{j}
\end{gathered}
$$

with

$$
\frac{\partial \bar{u}_{i}}{\partial x_{i}}=0
$$

where we used

$$
\overline{\left(\frac{\partial f}{\partial t}\right)}=\frac{\partial \bar{f}}{\partial t} \quad \text { and } \overline{\left(\frac{\partial f}{\partial x_{j}}\right)}=\frac{\partial \bar{f}}{\partial x_{j}}
$$

( $f$ being a dependent variable), and $\tau_{i j}$ is the so-called SGS stress tensor that generally needs to be modeled.

Before getting into the main subject, we present here some mathematical tools useful for the present investigation. Supposing that the Gaussian filter is applied in the $x_{i}$ direction, we have

$$
\overline{\left(x_{i} f\right)}=x_{i} \bar{f}+\frac{\Delta^{2}}{2 \gamma} \frac{\partial \bar{f}}{\partial x_{i}}
$$

and

$$
\overline{\left(x_{j} f\right)}=x_{j} \bar{f} \quad \text { for } j \neq i,
$$

which can be given in the same manner as shown in Refs. 12, 14, 15], and have been utilized in, e.g., Ref. [16]. Using Eq. (8), we have

$$
\begin{gathered}
\bar{x}_{i}=x_{i}, \\
\overline{\left(x_{i}^{2}\right)}=x_{i}^{2}+\frac{\Delta^{2}}{2 \gamma} .
\end{gathered}
$$

Based on these results, in the following subsections we perform theoretical investigations concerning the Gaussian filtered Navier-Stokes equations under a plane channel and swirling flow conditions.

\section{B. Plane channel flow}

Suppose that $x_{1}, x_{2}$, and $x_{3}$, respectively, are the streamwise, the wall-normal, and the spanwise directions, and a plane solid wall is set for $x_{2} \leq 0$. Decomposing the velocity components into the statistically averaged value $U_{i}$ and fluctuation part $u_{i}^{\prime}$, we have

$$
u_{i}(\mathbf{x}, t)=U_{i}(\mathbf{x})+u_{i}^{\prime}(\mathbf{x}, t),
$$

where $U_{i}$ is assumed to not depend on time. Furthermore, we assume that

$$
\begin{aligned}
\mathbf{U}(\mathbf{x}) & =\left(U_{1}(\mathbf{x}), U_{2}(\mathbf{x}), U_{3}(\mathbf{x})\right) \\
& =\left(\beta x_{2}, 0,0\right)
\end{aligned}
$$

with $\beta$ being a real constant, i.e., the mean streamwise velocity is linearly proportional to the distance from the wall. (The extension to more general cases is briefly discussed in Appendix A) Based on these assumptions, one knows

$$
\frac{\partial U_{i}}{\partial x_{i}}=\frac{\partial u_{i}^{\prime}}{\partial x_{i}}=0
$$

Substituting Eqs. (12) and (13) into the convection terms in the equation for $u_{1}$ and using Eq. (14), we have

$$
\begin{aligned}
\frac{\partial u_{j} u_{1}}{\partial x_{j}} & =U_{1} \frac{\partial u_{1}^{\prime}}{\partial x_{1}}+\frac{\partial U_{1}}{\partial x_{2}} u_{2}^{\prime}+\frac{\partial u_{j}^{\prime} u_{1}^{\prime}}{\partial x_{j}} \\
& =\beta x_{2} \frac{\partial u_{1}^{\prime}}{\partial x_{1}}+\beta u_{2}^{\prime}+\frac{\partial u_{j}^{\prime} u_{1}^{\prime}}{\partial x_{j}} .
\end{aligned}
$$

Appling Gaussian filtering in the wall-normal direction to this equation yields

$$
\begin{aligned}
\frac{\partial \overline{u_{j} u_{1}}}{\partial x_{j}} & =\bar{U}_{1} \frac{\partial \bar{u}_{1}^{\prime}}{\partial x_{1}}+\beta \frac{\Delta^{2}}{2 \gamma} \frac{\partial^{2} \bar{u}_{1}^{\prime}}{\partial x_{1} \partial x_{2}}+\frac{\partial \bar{U}_{1}}{\partial x_{2}} \bar{u}_{2}^{\prime}+\frac{\partial \overline{u_{j}^{\prime} u_{1}^{\prime}}}{\partial x_{j}} \\
& =\beta \bar{x}_{2} \frac{\partial \bar{u}_{1}^{\prime}}{\partial x_{1}}+\beta \frac{\Delta^{2}}{2 \gamma} \frac{\partial^{2} \bar{u}_{1}^{\prime}}{\partial x_{1} \partial x_{2}}+\beta \bar{u}_{2}^{\prime}+\frac{\partial \overline{u_{j}^{\prime} u_{1}^{\prime}}}{\partial x_{j}}
\end{aligned}
$$

where we use Eq. (8) and $U_{1}=\bar{U}_{1}$ given easily by Eq. (10). Even if a three-dimensional Gaussian filter is adopted, almost the same formula is derived because $U_{1}$ only depends on $x_{2}$, and hence no additional term is derived by filtering in the other directions. Meanwhile, the convection terms in terms of the filtered velocity components, $\partial\left(\bar{u}_{j} \bar{u}_{1}\right) / \partial x_{j}$, can be written as

$$
\frac{\partial \bar{u}_{j} \bar{u}_{1}}{\partial x_{j}}=\bar{U}_{1} \frac{\partial \bar{u}_{1}^{\prime}}{\partial x_{1}}+\frac{\partial \bar{U}_{1}}{\partial x_{2}} \bar{u}_{2}^{\prime}+\frac{\partial \bar{u}_{j}^{\prime} \bar{u}_{1}^{\prime}}{\partial x_{j}} .
$$

From Eqs. (16) and (17), we have

$$
\frac{\partial \tau_{1 j}}{\partial x_{j}}=\beta \frac{\Delta^{2}}{2 \gamma} \frac{\partial^{2} \bar{u}_{1}^{\prime}}{\partial x_{1} \partial x_{2}}+\frac{\partial\left(\overline{u_{j}^{\prime} u_{1}^{\prime}}-\bar{u}_{j}^{\prime} \bar{u}_{1}^{\prime}\right)}{\partial x_{j}} .
$$

Substituting this and $\partial^{2} \bar{U}_{1} / \partial x_{j} \partial x_{j}=0$ into Eq. (6) yields

$$
\begin{aligned}
\frac{\partial \bar{u}_{1}}{\partial t}+\frac{\partial \bar{u}_{j} \bar{u}_{1}}{\partial x_{j}}= & -\frac{\partial \bar{p}}{\partial x_{1}}+\nu \frac{\partial^{2} \bar{u}_{1}^{\prime}}{\partial x_{j} \partial x_{j}}-\beta \frac{\Delta^{2}}{2 \gamma} \frac{\partial^{2} \bar{u}_{1}^{\prime}}{\partial x_{1} \partial x_{2}} \\
& -\frac{\partial\left(\overline{u_{j}^{\prime} u_{1}^{\prime}}-\bar{u}_{j}^{\prime} \bar{u}_{1}^{\prime}\right)}{\partial x_{j}}
\end{aligned}
$$


which can be considered an equation for both $\bar{u}_{1}$ and $\bar{u}_{1}^{\prime}$ because

$$
\frac{\partial \bar{u}_{1}}{\partial t}=\frac{\partial \bar{u}_{1}^{\prime}}{\partial t}
$$

The second-to-the-last term of Eq. (19) is a crossderivative of the dependent variable $\bar{u}_{1}^{\prime}$. As has been proved in several studies (e.g., Refs. 12, 15]), the derivatives of this type should be numerically unstable when, for instance, a finite difference technique is adopted for solving the equation. In what follows, we consider the stability condition for Eq. (19).

The last three terms of Eq. (19) may make a dominant contribution to the numerical stability of LES. We will first focus our attention on the second- and third-to-thelast terms:

$$
\nu \frac{\partial^{2} \bar{u}_{1}^{\prime}}{\partial x_{j} \partial x_{j}}-\beta \frac{\Delta^{2}}{2 \gamma} \frac{\partial^{2} \bar{u}_{1}^{\prime}}{\partial x_{1} \partial x_{2}}
$$

Appling the coordinate transformation of

$$
(\xi, \eta)=\left(\frac{x_{1}+x_{2}}{\sqrt{2}}, \frac{-x_{1}+x_{2}}{\sqrt{2}}\right),
$$

which represents rotation by $45^{\circ}$ around the $x_{3}$ axis, Eq. (20) is rewritten as

$$
\nu\left(\frac{\partial^{2} \bar{u}_{1}^{\prime}}{\partial \xi^{2}}+\frac{\partial^{2} \bar{u}_{1}^{\prime}}{\partial \eta^{2}}+\frac{\partial^{2} \bar{u}_{1}^{\prime}}{\partial x_{3}^{2}}\right)-\beta \frac{\Delta^{2}}{2 \gamma}\left(\frac{1}{2} \frac{\partial^{2} \bar{u}_{1}^{\prime}}{\partial \xi^{2}}-\frac{1}{2} \frac{\partial^{2} \bar{u}_{1}^{\prime}}{\partial \eta^{2}}\right) .
$$

From this, it can be seen that the following restriction is required to ensure that Eq. (22) is a positive viscosity:

$$
\nu-\frac{\Delta^{2}}{4 \gamma}|\beta| \geq 0
$$

The shear gradient $\beta$ may have different values depending on the problems considered. We consider here a simple example to show concretely how restriction (23) works in a realistic situation. As is well known, in the viscous sublayer of turbulent channel flows, the statistically averaged streamwise velocity follows 17]

$$
U_{1}\left(x_{2}\right)=u_{\tau}\left(\frac{\left|u_{\tau}\right|}{\nu} x_{2}\right)
$$

with $u_{\tau}$ being the wall-friction velocity. Using Eq. (24) and rewriting reduce Eq. (23) to

$$
\frac{\left|u_{\tau}\right|}{\nu} \Delta=\Delta^{+} \leq 2 \sqrt{\gamma}
$$

where the superscript + denotes a quantity in the wall units. For $\gamma=6$, this result essentially corresponds to the stability condition derived by Kobayashi and Shimomura from the tensor-diffusivity term in a dynamic SGS model 11.

We should note here that Eq. (25) is a minimum condition for stabilizing the numerical solution; the last term of Eq. (19), which we have neglected, can also be a seed of numerical instability because if, for example, $u_{1}^{\prime} \propto x_{2}$ is true at a certain instant and location, then

$$
\overline{\left(u_{1}^{\prime} \frac{\partial u_{1}^{\prime}}{\partial x_{1}}\right)}-\bar{u}_{1}^{\prime} \frac{\partial \bar{u}_{1}^{\prime}}{\partial x_{1}} \propto \frac{\partial^{2} \bar{u}_{1}^{\prime}}{\partial x_{1} \partial x_{2}} .
$$

(See Ref. [12].) Nevertheless, this incomplete condition creates a strong restriction on the grid width $(h)$. The grid width should satisfy $h^{+} \leq \sqrt{6} \approx 2.4$ for $\Delta^{+}=2 h^{+}$, a setting that has frequently been used, or $h^{+} \leq \sqrt{6} / 2 \approx$ 1.2 for $\Delta^{+}=4 h^{+}$, which is necessary for the contribution of the SGS force to be significant compared to the truncation and aliasing errors of a second-order finite difference scheme [18]. (We should note here that this restriction is imposed only on the grid width in the wall-normal direction because only the filtering in this direction yields the cross-derivative term, as mentioned already.) This result implies that at least several grid points are needed in the viscous sublayer to guarantee numerical stability, which is a formidable restriction in practical applications of a large-scale high-Reynolds-number turbulent flow. It is known that when the inner layer of turbulent boundarylayer flows is resolved, the number of grid points required for LES exceeds current computational capacities even at moderate Reynolds numbers, and hence developments of SGS models that are applicable to a coarse wall-normal grid and of wall models that do not need a strong refinement of the near-wall grids are the most pressing issues of LES; see a recent review by Piomelli and Balaras [19]. The numerical problem that we have suggested above provides a taste of the difficulties of constructing an excellent model.

The stability condition presented above would be partly weakened if the last term of Eq. (19) is modeled using an eddy-viscosity model. However, this treatment does not always achieve a stable solution. Actually, Winckelmans et al. [8] have observed by numerical experiments that for a plane channel flow, a dynamic mixed model based on the tensor-diffusivity model (which can accurately represent the characteristics of second-order cross derivatives [11]) generates numerical instabilities that eventually make the numerical simulation blow up. To remove those instabilities, they were forced to add an artificial damping coefficient to the tensor-diffusivity portion. In the mixed model that they used, the coefficient of eddy-viscosity part was well tuned by a dynamic procedure. Essentially the same observations of the numerical instability of the dynamic mixed model can be found in Ref. [11] by Kobayashi and Shimomura. In those studies, the wall-normal grid width was set to be several times larger than that used in DNS. That is, those studies suggest that, for a large grid (and filter) width, which is much-needed in practical applications, the addition of an eddy-viscosity term does not always guarantee numerical stability. If, on the other hand, all the SGS terms are modeled using an eddy-viscosity model whose coefficient is guaranteed to be positive, the numerical stability of the filtered system would be guaran- 
teed in many cases. However, it has been suggested by many researchers that eddy-viscosity models have several deficiencies that make the LES results suspicious (e.g., Refs. 2, 9, 20, 21, 22, 23, 24, 25]). For instance, as has frequently been demonstrated by a priori tests using DNS techniques, the SGS quantities modeled using eddy-viscosity models do not correlate well with the actual SGS quantities [2, 9, 20, 23, 26], implying that these models have low accuracy and/or narrow applicability. Indeed, in the present case the eddy-viscosity models cannot describe the cross-derivative term in Eq. (19), which is a hybrid of positive and negative diffusions. Moreover, eddy-viscosity models were constructed under the assumptions that the subgrid turbulence is isotropic and the filter scale is in an inertial range, assumptions that are in general both violated in inhomogeneous turbulent flows, especially near walls. Furthermore, the dynamic types of eddy-viscosity models need artificial techniques for smoothing the model coefficient and for clipping its negative values [2, 5, 6, 7, 27]. Based on the above, we consider the eddy-viscosity models to be insufficient. Most of the recent efforts at SGS modeling have been devoted to developing a model that does not strongly rely on eddy-viscosity models 7, 8, 25, 26, 28, 29, 30, 31]. Models based on a kind of "defiltering" procedure [25, 29, 30] are one such approach, determining significant portions of the SGS terms by an analytical, not empirical, procedure. The mixed models based on the tensor-diffusivity term [7, 8, 31] can also be categorized into this type [26]. We should note here that attempts to improve eddy-viscosity models are also being continued; see, e.g., Refs. [24, 27, 32, 33].

It is worth noting that the numerical stability of the cross-derivative term in Eq. (19) is time-independent, as this term always reveals a negative diffusivity in a fixed range of directions, whereas the numerical stability of the last term of the same equation may be time-dependent. As mentioned in Sec. 1] Winckelmans et al. have pointed out that the numerical stability of the tensor-diffusivity model depends on the problems that they have considered; obtaining stable results is difficult to achieve for a strongly inhomogeneous (wall-bounded) flow, even when an eddy-viscosity term is added, but is possible for a homogeneous flow even by the pure tensor-diffusivity model 8]. They have explained these results as follows: For the homogeneous flow, the direction in which the tensordiffusivity term reveals the negative diffusivity evolves continuously in time and space, and hence the model term may be dissipative on time average. However, in the inhomogeneous flow case, long-lived negative diffusion events can take place near the wall, leading to numerical instability and a resulting divergence of the solution. As mentioned above, those numerical findings indicate an inadequacy of the previous stability analyses by Leonard [10] and by Ida and Taniguchi [12], which have only considered the instantaneous behavior of the SGS model or the SGS term. The present theoretical findings, showing the appearance of a term that is always numerically unstable in fixed directions and hence is unstable on time-average as well, can more correctly explain the problematic numerical instability encountered in the simulations of plane channel flows [8, 11].

\section{Swirling flow}

Turbulent flows involving a large-scale swirl are of practical importance in connection with combustion engineering, aeroacoustics, meteorology, and so on. In this subsection, we discuss the LES of turbulent flows having a rotating mean velocity about the $x_{3}$ (or $z$ ) axis, and show that the existence of the swirl can cause numerical instability through the Gaussian filtering operation.

First, we consider a very simple case in which the swirl has a constant angular velocity; that is, the mean velocity takes the form of

$$
\mathbf{U}(\mathbf{x})=\left(\alpha x_{2},-\alpha x_{1}, 0\right)
$$

where $\alpha$ is a real constant. This mean velocity satisfies

$$
\begin{gathered}
\mathbf{U} \cdot \mathbf{x}^{*}=0, \\
|\mathbf{U}| /\left|\mathbf{x}^{*}\right|=|\alpha|,
\end{gathered}
$$

and

$$
\frac{\partial U_{i}}{\partial x_{i}}=0
$$

where $\mathbf{x}^{*} \equiv\left(x_{1}, x_{2}, 0\right)$. As a result, we know

$$
\frac{\partial u_{i}^{\prime}}{\partial x_{i}}=0
$$

Using the above, the convection terms for $u_{1}$ are rewritten as

$$
\frac{\partial u_{j} u_{1}}{\partial x_{j}}=U_{1} \frac{\partial u_{1}^{\prime}}{\partial x_{1}}+U_{2} \frac{\partial u_{1}^{\prime}}{\partial x_{2}}+\frac{\partial U_{1}}{\partial x_{2}} u_{2}^{\prime}+U_{2} \frac{\partial U_{1}}{\partial x_{2}}+\frac{\partial u_{j}^{\prime} u_{1}^{\prime}}{\partial x_{j}},
$$

where the first four terms on the right-hand side (RHS) result from the existence of the swirl, among which the first and second terms,

$$
U_{1} \frac{\partial u_{1}^{\prime}}{\partial x_{1}}+U_{2} \frac{\partial u_{1}^{\prime}}{\partial x_{2}}=\alpha x_{2} \frac{\partial u_{1}^{\prime}}{\partial x_{1}}-\alpha x_{1} \frac{\partial u_{1}^{\prime}}{\partial x_{2}},
$$

may have a significant influence on the numerical stability of the equation for $\bar{u}_{1}^{\prime}$.

We show here that the filtered formula and its stability change depending on how the Gaussian filtering is applied. Appling the Gaussian filter in the $x_{2}$ direction to Eq. (29) results in

$$
\overline{\left(U_{1} \frac{\partial u_{1}^{\prime}}{\partial x_{1}}\right)}+\overline{\left(U_{2} \frac{\partial u_{1}^{\prime}}{\partial x_{2}}\right)}=\alpha x_{2} \frac{\partial \bar{u}_{1}^{\prime}}{\partial x_{1}}+\alpha \frac{\Delta^{2}}{2 \gamma} \frac{\partial^{2} \bar{u}_{1}^{\prime}}{\partial x_{1} \partial x_{2}}-\alpha x_{1} \frac{\partial \bar{u}_{1}^{\prime}}{\partial x_{2}}
$$


which can be rewritten as

$$
\overline{\left(U_{1} \frac{\partial u_{1}^{\prime}}{\partial x_{1}}\right)}-\bar{U}_{1} \frac{\partial \bar{u}_{1}^{\prime}}{\partial x_{1}}+\overline{\left(U_{2} \frac{\partial u_{1}^{\prime}}{\partial x_{2}}\right)}-\bar{U}_{2} \frac{\partial \bar{u}_{1}^{\prime}}{\partial x_{2}}=\alpha \frac{\Delta^{2}}{2 \gamma} \frac{\partial^{2} \bar{u}_{1}^{\prime}}{\partial x_{1} \partial x_{2}} .
$$

The cross-derivative term appearing on the RHS may cause numerical instability when the absolute value of its coefficient is sufficiently large. In contrast, when the Gaussian filter is applied in both the $x_{1}$ and $x_{2}$ directions, we obtain a stable formula,

$$
\begin{aligned}
\overline{\left(U_{1} \frac{\partial u_{1}^{\prime}}{\partial x_{1}}\right)}+\overline{\left(U_{2} \frac{\partial u_{1}^{\prime}}{\partial x_{2}}\right)}= & \alpha x_{2} \frac{\partial \bar{u}_{1}^{\prime}}{\partial x_{1}}+\alpha \frac{\Delta^{2}}{2 \gamma} \frac{\partial^{2} \bar{u}_{1}^{\prime}}{\partial x_{1} \partial x_{2}} \\
& -\alpha x_{1} \frac{\partial \bar{u}_{1}^{\prime}}{\partial x_{2}}-\alpha \frac{\Delta^{2}}{2 \gamma} \frac{\partial^{2} \bar{u}_{1}^{\prime}}{\partial x_{1} \partial x_{2}} \\
= & \alpha x_{2} \frac{\partial \bar{u}_{1}^{\prime}}{\partial x_{1}}-\alpha x_{1} \frac{\partial \bar{u}_{1}^{\prime}}{\partial x_{2}}
\end{aligned}
$$

and thus,

$$
\overline{\left(U_{1} \frac{\partial u_{1}^{\prime}}{\partial x_{1}}\right)}-\bar{U}_{1} \frac{\partial \bar{u}_{1}^{\prime}}{\partial x_{1}}+\overline{\left(U_{2} \frac{\partial u_{1}^{\prime}}{\partial x_{2}}\right)}-\bar{U}_{2} \frac{\partial \bar{u}_{1}^{\prime}}{\partial x_{2}}=0 .
$$

Equation (31) has no cross derivative.

Next, we consider the same example but in the cylindrical coordinate $(r, \theta, z)$. In this case, the mean velocity (26) and the instantaneous velocity, respectively, are represented by

$$
\mathbf{U}(r, \theta, z)=\left(U_{r}, U_{\theta}, U_{z}\right)=(0,-\alpha r, 0),
$$

and

$$
\left(u_{r}, u_{\theta}, u_{z}\right)=\left(u_{r}^{\prime}, U_{\theta}+u_{\theta}^{\prime}, u_{z}^{\prime}\right),
$$

and the nonlinear terms in the Navier-Stokes equation for $u_{\theta}$ take the form of

$$
u_{r} \frac{\partial u_{\theta}}{\partial r}+\frac{u_{\theta}}{r} \frac{\partial u_{\theta}}{\partial \theta}+u_{z} \frac{\partial u_{\theta}}{\partial z}+\frac{u_{r} u_{\theta}}{r} \equiv h_{\theta},
$$

where the velocity components should satisfy

$$
\begin{aligned}
0 & =\frac{\partial u_{r}}{\partial r}+\frac{u_{r}}{r}+\frac{1}{r} \frac{\partial u_{\theta}}{\partial \theta}+\frac{\partial u_{z}}{\partial z} \\
& =\frac{\partial u_{r}^{\prime}}{\partial r}+\frac{u_{r}^{\prime}}{r}+\frac{1}{r} \frac{\partial u_{\theta}^{\prime}}{\partial \theta}+\frac{\partial u_{z}^{\prime}}{\partial z} .
\end{aligned}
$$

Substituting Eq. (33) into Eq. (34) yields

$$
\begin{aligned}
h_{\theta}= & \frac{U_{\theta}}{r} \frac{\partial u_{\theta}^{\prime}}{\partial \theta}+\left(\frac{\partial U_{\theta}}{\partial r}+\frac{U_{\theta}}{r}\right) u_{r}^{\prime} \\
& +\left(u_{r}^{\prime} \frac{\partial u_{\theta}^{\prime}}{\partial r}+\frac{u_{\theta}^{\prime}}{r} \frac{\partial u_{\theta}^{\prime}}{\partial \theta}+u_{z}^{\prime} \frac{\partial u_{\theta}^{\prime}}{\partial z}+\frac{u_{r}^{\prime} u_{\theta}^{\prime}}{r}\right) .
\end{aligned}
$$

The terms in the last parentheses represent the nonlinear interaction between the fluctuation components, whereas the remaining terms describe the interaction between the mean and fluctuation portions. Appling the Gaussian filter in the $r$ direction with a filter width of $\Delta_{r}$ to the first term results in

$$
\overline{\left(\frac{U_{\theta}}{r} \frac{\partial u_{\theta}^{\prime}}{\partial \theta}\right)}=-\alpha \frac{\partial \bar{u}_{\theta}^{\prime}}{\partial \theta}=\frac{\bar{U}_{\theta}}{r} \frac{\partial \bar{u}_{\theta}^{\prime}}{\partial \theta},
$$

where we used Eq. (10) and assumed $r \gg \Delta_{r}$ so that the value of the Gaussian filter is sufficiently close to zero at $r=0$. No unstable term appears in Eq. (37). Even when the Gaussian filter is applied in both the $r$ and $\theta$ directions, almost the same formula is obtained because $U_{\theta}$ depends only on $r$. Specifically, in the present case the resulting formula is not, unlike the previous case, dependent on how the Gaussian filter is applied.

The above results, which reveal that the numerical stability of LES depends not only on the flow configuration but also on the filtering strategy and coordinate system adopted, can be interpreted as follows: As has been shown in Ref. 12] and in the preceding subsection, the existence of a shear leads to the appearance of a numerically unstable term. Although no shear exists in the mean flow profile described by Eq. (26), a virtual shear is observed when the Gaussian filter is applied only in one direction in the Cartesian coordinate $\left(x_{1}\right.$ or $\left.x_{2}\right)$, resulting in the derivation of the cross-derivative term. This problem does not arise for the cylindrical coordinate, since the mean convection velocity in the $(r, \theta)$ space,

$$
\left(\frac{d r}{d t}, \frac{d \theta}{d t}\right)=\left(U_{r}, \frac{U_{\theta}}{r}\right)=(0,-\alpha),
$$

is uniform.

Lastly, we consider a case where the mean velocity has a second-order term, i.e., assuming

$$
\mathbf{U}(r, \theta, z)=\left(0, \alpha_{1} r+\alpha_{2} r^{2}, 0\right),
$$

where $\alpha_{1}$ and $\alpha_{2}$ are real constants. Substituting this into the first term on the RHS of Eq. (36) and applying the Gaussian filter in the $r$ direction, we have

$$
\overline{\left(\frac{U_{\theta}}{r} \frac{\partial u_{\theta}^{\prime}}{\partial \theta}\right)}=\left(\alpha_{1}+\alpha_{2} r\right) \frac{\partial \bar{u}_{\theta}^{\prime}}{\partial \theta}+\alpha_{2} \frac{\Delta_{r}^{2}}{2 \gamma} \frac{\partial^{2} \bar{u}_{\theta}^{\prime}}{\partial r \partial \theta},
$$

which can be rewritten as

$$
\overline{\left(\frac{U_{\theta}}{r} \frac{\partial u_{\theta}^{\prime}}{\partial \theta}\right)}-\frac{\bar{U}_{\theta}}{r} \frac{\partial \bar{u}_{\theta}^{\prime}}{\partial \theta}=-\alpha_{2} \frac{\Delta_{r}^{2}}{2 \gamma r} \frac{\partial \bar{u}_{\theta}^{\prime}}{\partial \theta}+\alpha_{2} \frac{\Delta_{r}^{2}}{2 \gamma} \frac{\partial^{2} \bar{u}_{\theta}^{\prime}}{\partial r \partial \theta}
$$

because, based on Eqs. (10) and (11),

$$
\bar{U}_{\theta}=\alpha_{1} r+\alpha_{2}\left(r^{2}+\frac{\Delta_{r}^{2}}{2 \gamma}\right) .
$$

The last term of Eq. (40) is a cross derivative of the dependent variable and should destabilize the numerical solution, whereas the second-to-the-last term represents convection in the $\theta$ direction that can be solved stably. For $\alpha_{2}=0$, the RHS terms of Eq. (40) vanish, and this equation corresponds to Eq. (37). More specifically, the 
instability in this case is due to the second-order component in the mean velocity, which violates the uniformity of the angular velocity and thus represents a shear.

As in the plane-channel case, the cross-derivative terms derived above have a time-independent coefficient and thus should lead to numerical instability if an unsuitably large filter is adopted. The present results imply that such an unstable term can appear in many situations. In a future paper, we will consider more general and complicated flow configurations to create a generalized theory of numerical instability due to filtering operations [34].

\section{SUMMARY AND CONCLUSION}

In summary, we have theoretically investigated the numerical instability of LES caused by the filtering procedure based on simple assumptions regarding the statistically averaged velocity, and have shown that Gaussian filtering yields a numerically unstable term, which always reveals a negative diffusivity in a fixed range of directions in cases of both plane channel flow and swirling flow. This conclusion confirms and extends that of our previous work [12]. We anticipate that it would be interesting to examine the relationship between this numerical instability and the shear-induced (physical) instabilities in turbulence [35, 36].

Furthermore, we have presented several additional findings. In Sec. IIB we showed that in channelflow case, this numerical problem strongly restricts the wall-normal grid width necessary for achieving stable and accurate solutions. In the academic computations of a plane channel flow using an LES technique, the grid width in the wall-normal direction has sometimes been set to almost the same as that required in DNS, and no filter is adopted in this direction (e.g., Refs. [5, 20, 21, 28, 32]); the present result provides a grounding for this custom. Furthermore, this result indicates a significant difficulty in developing an excellent SGS model that can provide satisfactory (i.e., not only stable but also accurate) solutions, even with large wallnormal filter widths, which is seriously desired in practical applications of high- (and even moderate-) Reynoldsnumber turbulence. In Sec. IIC based on investigations of swirling flow cases, we pointed out that the numerical stability of the filtered equations depends not only on the flow configuration, but also on the dimension of the Gaussian filtering and the adopted coordinate system. This finding would be of particular importance in practical applications that use a general curvilinear coordinate system or unstructured grids.

In the present study (and also in our previous paper), it was assumed implicitly that all the spectral modes contained in the velocity field are fully resolved because the Gaussian filter does not cut off any modes, but only damps the high-frequency modes. (See Appendix B which presents a related remark concerning cases where the spectral cutoff or the top-hat filter is employed, both of which eliminate certain modes.) The present results therefore indicate that even in such an ideal case, the complete SGS model (or, more precisely, the complete subfilter-scale model whose characteristic length is determined independently from the grid width), which can completely reproduce the properties of real SGS stresses, can be numerically unstable when treated numerically, since the complete model perfectly describes the characteristics of the unstable cross-derivative term. This problem poses a dilemma for practitioners of LES who are looking for an accurate and stable SGS model. To improve model accuracy, this numerical instability problem should be confronted. However, if a rough, artificial treatment is adopted for stability, the accuracy of the LES results will not be guaranteed. A potential approach to overcoming this difficulty is, as suggested in our previous paper 12], to construct a stable and accurate numerical solver for the numerically unstable term, though this would admittedly be quite a difficult task. (Leonard [10] and Moeleker and Leonard [37] have proposed Lagrangian methods based on the tensor-diffusivity model and an anisotropic particle scheme to solve the negativediffusion problems, and have achieved stable solutions for a two-dimensional scalar transport equation with known velocity fields. However, several issues remain to be overcome to extend those methods to the case of NavierStokes turbulence [37].) Further careful and vigorous considerations are necessary for this instability problem to be solved.

\section{Acknowledgments}

One of the authors (M.I.) thanks T. Tominaga, M. Miyazawa, and K. Matsuura for their valuable comments. This work was supported by the Ministry of Education, Culture, Sports, Science, and Technology of Japan (Monbu-Kagaku-Sho) under an IT research program "Frontier Simulation Software for Industrial Science."

\section{APPENDIX A: CLOSURE FOR HIGH-ORDER MEAN VELOCITY}

As a first step toward constructing a general theory for the filtering instability, let us consider the closure of

$$
\overline{\left(U_{1} \frac{\partial u_{1}^{\prime}}{\partial x_{1}}\right)}
$$

in the case where $U_{1}$ is a general function of $x_{2}$, under the assumption that the Gaussian filter is applied in the $x_{2}$ direction. Using the Taylor expansion, the arbitrary function $U_{1}\left(x_{2}\right)$ is represented by a polynomial of infinite order:

$$
U_{1}\left(x_{2}\right)=\sum_{n=0}^{\infty} a_{n} x_{2}^{n},
$$


where $a_{n}$ are real constants. Therefore, what we have to do is to consider the closure of

$$
\overline{\left(x_{2}^{n} \frac{\partial u_{1}^{\prime}}{\partial x_{1}}\right)}
$$

with $n$ being an arbitrary positive integer. This aim is achieved as follows: Using Eq. (8) successively, Eq. (A1) is rewritten into a closed form in terms of $\bar{u}_{1}^{\prime}$, as

$$
\begin{aligned}
\overline{\left(x_{2}\left(x_{2}^{n-1} \frac{\partial u_{1}^{\prime}}{\partial x_{1}}\right)\right)} & =\chi \overline{\left(x_{2}^{n-1} \frac{\partial u_{1}^{\prime}}{\partial x_{1}}\right)} \\
& =\chi \chi \overline{\left(x_{2}^{n-2} \frac{\partial u_{1}^{\prime}}{\partial x_{1}}\right)} \\
\cdots & =\chi^{n} \frac{\partial \bar{u}_{1}^{\prime}}{\partial x_{1}}
\end{aligned}
$$

where

$$
\chi \equiv x_{2}+\frac{\Delta^{2}}{2 \gamma} \frac{\partial}{\partial x_{2}} .
$$

The resulting formula has derivatives of high orders, and hence stability analysis would be somewhat intricate.

\section{APPENDIX B: SPECTRAL CUTOFF AND TOP-HAT FILTERS}

In Ref. 14], Klimas showed that the Gaussian filtered Vlasov equation can be rewritten into a closed form in terms of the filtered distribution function without any approximation. Also, in Ref. [38] (see also Refs. 10, 26]) Yeo showed that the Gaussian filtered Navier-Stokes equations are written in a closed form having an infinite series. Their results have been utilized in our study, as described in the present and previous papers. This Appendix is devoted to showing that when the spectral cutoff or the top-hat filter is adopted, it is in general impossible to analytically derive an exact, closed formula.

Let us consider two arbitrary velocity fields

$$
\mathbf{u}^{(m)}(\mathbf{x})=\left(u_{1}^{(m)}(\mathbf{x}), u_{2}^{(m)}(\mathbf{x}), u_{3}^{(m)}(\mathbf{x})\right) \quad \text { for } m=1,2,
$$

whose spectra are

$$
\hat{\mathbf{u}}^{(m)}(\mathbf{k})=\left(\hat{u}_{1}^{(m)}(\mathbf{k}), \hat{u}_{2}^{(m)}(\mathbf{k}), \hat{u}_{3}^{(m)}(\mathbf{k})\right) \quad \text { for } m=1,2,
$$

where $\mathbf{x}=\left(x_{1}, x_{2}, x_{3}\right)$ and $\mathbf{k}$ is the wavenumber vector. If these velocities, at a certain instant $t_{1}$, satisfy

$$
\hat{\mathbf{u}}^{(1)}(\mathbf{k}) \neq \hat{\mathbf{u}}^{(2)}(\mathbf{k}) \quad \text { for }|\mathbf{k}|>k_{c}
$$

but

$$
\hat{\mathbf{u}}^{(1)}(\mathbf{k})=\hat{\mathbf{u}}^{(2)}(\mathbf{k}) \text { for }|\mathbf{k}| \leq k_{c},
$$

and

$$
\overline{\mathbf{u}^{(1)}}(\mathbf{x})=\overline{\mathbf{u}^{(2)}}(\mathbf{x}) \text { for all } \mathbf{x},
$$

where $\overline{(\cdot)}$ denotes the spectral cutoff with an identical cutoff wavenumber $k_{c}$, then the closed equation will provide the same result after this instant [i.e., $\overline{\mathbf{u}^{(1)}}(\mathbf{x}, t)=$ $\overline{\mathbf{u}^{(2)}}(\mathbf{x}, t)$ for $\left.t>t_{1}\right]$, although the unfiltered velocities have different high-wavenumber modes. This unacceptable conclusion has resulted from the assumption that a closed formula exists.

If, on the other hand,

$$
\mathbf{u}^{(1)}(\mathbf{x})=\mathbf{u}^{(2)}(\mathbf{x})+\cos \left(k x_{2}\right) \mathbf{i}
$$

is true at an instant (where $k$ is a constant wavenumber and $\mathbf{i}$ denotes the unit vector in the $x_{1}$ direction) and the wavelength of the cosine function in this equation is, for example, equal to the filter width of the top-hat filter applied (at least) in the $x_{2}$ direction, then

$$
\overline{\mathbf{u}^{(1)}}(\mathbf{x})=\overline{\mathbf{u}^{(2)}}(\mathbf{x}) \text { for all } \mathbf{x} .
$$

(Here, $\overline{(\cdot)}$ denotes the top-hat filtering.) As in the previous case, this result denies the existence of a closed formula.

The above results imply that in order for an exact closed formula to exist, the spectrum of the applied filter needs to have a nonzero value for $|\mathbf{k}| \neq \infty$. The convolution product in the physical space using a filter function $L$,

$$
\overline{\mathbf{u}^{(m)}}(\mathbf{x})=\int_{-\infty}^{\infty} L(\mathbf{x}-\xi) \mathbf{u}^{(m)}(\xi) d \xi
$$

is represented in the Fourier space by the simple multiplication of the spectra of $L$ and $\mathbf{u}^{(m)}$ :

$$
\overline{\hat{\mathbf{u}}^{(m)}}(\mathbf{k})=\hat{L}(\mathbf{k}) \hat{\mathbf{u}}^{(m)}(\mathbf{k}) \text {. }
$$

Based on the above, we know that if $\hat{\mathbf{u}}^{(1)}(\mathbf{k}) \neq \hat{\mathbf{u}}^{(2)}(\mathbf{k})$ for certain $\mathbf{k}$ and $\hat{L}(\mathbf{k})$ has a nonzero value for all $\mathbf{k}$, then $\overline{\hat{\mathbf{u}}^{(1)}}(\mathbf{k}) \neq \overline{\hat{\mathbf{u}}^{(2)}}(\mathbf{k})$ at least for the certain $\mathbf{k}$ and consequently $\overline{\mathbf{u}^{(1)}}(\mathbf{x}) \neq \overline{\mathbf{u}^{(2)}}(\mathbf{x})$ at least in some regions; more specifically, if the adopted filter function is not equal to zero for all $\mathbf{k}$, filtering different velocity fields results in different filtered velocity fields.

The present conclusion is in opposition to the theoretical result of Carati et al. 26] which proved mathematically the existence of a closed formula in the top-hat case. Let us consider the cause of this discrepancy. In the study of Carati et al., it was first assumed that the generalized expansion series for one-dimensional filtering in the $x$ direction takes the form of

$$
\overline{(a b)}=\sum_{r, s=0}^{\infty} c_{r s} \partial_{x}^{r} \bar{a} \partial_{x}^{s} \bar{b},
$$

where $a$ and $b$ are arbitrary, continuous, and differentiable functions of $x$ and $c_{r s}$ are real constants. We show here that this assumption is not always valid for top-hat 
filters. If $a$ is a sinusoidal wave whose wavelength is equal to the characteristic width of the applied top-hat filter, then $\bar{a}(x)=0$ for any value of $x$ and consequently the right-hand side of Eq. (B1) is also equal to zero for any $x$ if $c_{r s}$ are finite values. However, the left-hand side of this equation is not necessarily zero under the present condition; if, for example, $b=a$, then $a b \geq 0$ for any $x$ and thus $\overline{(a b)}>0$. This contradiction is caused by the fact that the value of the top-hat filters can be zero in the Fourier space. The generating function, Eq. (3.7) of Ref. [26] used to derive the generalized expansion series, is definable only for the filters that always have a nonzero value in the Fourier space as Gaussian filters, because that function diverges at the wavenumbers for which the filter value is zero. Therefore, the expansion series for top-hat filters is definable only if the filter width is smaller than the smallest resolved scale. When this restriction is not fulfilled, the expansion series should not converge.
[1] J. L. Lumley and A. M. Yaglom, Flow Turbul. Combust. 66, 241 (2001); Y. G. Sinai, Physica A 263, 565 (1999).

[2] C. Meneveau and J. Katz, Annu. Rev. Fluid Mech. 32, 1 (2000).

[3] A. Leonard, Adv. Geophys. 18, 237 (1974).

[4] J. Bardina, J. H. Ferziger, and W. C. Reynolds, AIAA Pap. No. 80-1357 (1980).

[5] M. Germano, U. Piomelli, P. Moin, and W. H. Cabot, Phys. Fluids A 3, 1760 (1991).

[6] R. Akhavan, A. Ansari, S. Kang, and N. Mangiavacchi, J. Fluid Mech. 408, 83 (2000).

[7] B. Vreman, B. Geurts, and H. Kuerten, J. Fluid Mech. 339, 357 (1997).

[8] G. S. Winckelmans, A. A. Wray, O. V. Vasilyev, and H. Jeanmart, Phys. Fluids 13, 1385 (2001).

[9] S. Liu, C. Meneveau, and J. Katz, J. Fluid Mech. 275, 83 (1994).

[10] A. Leonard, AIAA Pap. No. 97-0204 (1997).

[11] H. Kobayashi and Y. Shimomura, Phys. Fluids 15, L29 (2003).

[12] M. Ida and N. Taniguchi, Phys. Rev. E 68, 036705 (2003).

[13] P. Sagaut, Large Eddy Simulation for Incompressible Flows, 2nd edition (Springer-Verlag, Berlin, New York, Heidelberg, 2002).

[14] A. J. Klimas, J. Comput. Phys. 68, 202 (1987).

[15] H. Figua, F. Bouchut, M. R. Feix, and E. Fijalkow, J. Comput. Phys. 159, 440 (2000).

[16] Horiuti, in Recent Advances in DNS and LES, edited by D. Knight and L. Sakell (Kluwer Academic, Dordrecht, 1999), p. 179; Y. Shimomura, J. Phys. Soc. Jpn. 68, 2483 (1999).

[17] H. Tennekes and J. L. Lumley, A First Course in Turbulence (The MIT Press, Cambridge, MA, 1972).

[18] S. Ghosal, J. Comput. Phys. 125, 187 (1996); F. K. Chow and P. Moin, J. Comput. Phys. 184, 366 (2003).

[19] U. Piomelli and E. Balaras, Annu. Rev. Fluid Mech. 34,
349 (2002).

[20] K. Horiuti, Phys. Fluids A 1, 426 (1989); K. Horiuti, J. Phys. Soc. Jpn. 66, 91 (1997).

[21] S. Völker, R. D. Moser, and P. Venugopal, Phys. Fluids 14, 3675 (2002).

[22] J. A. Langford and R. D. Moser, J. Fluid Mech. 398, 321 (1999).

[23] R. A. Clark, J. H. Ferziger, and W. C. Reynolds, J. Fluid Mech. 91, 1 (1979).

[24] F. Porté-Agel, C. Meneveau, and M. B. Parlange, J. Fluid Mech. 415, 261 (2000).

[25] J. A. Domaradzki and K.-C. Loh, in Recent Advances in $D N S$ and LES, edited by D. Knight and L. Sakell (Kluwer Academic, Dordrecht, 1999), p. 121.

[26] D. Carati, G. S. Winckelmans, and H. Jeanmart, J. Fluid Mech. 441, 119 (2001).

[27] J. G. M. Kuerten, B. J. Geurts, A. W. Vreman, and M. Germano, Phys. Fluids 11, 3778 (1999).

[28] F. Sarghini, Piomelli, and E. Balaras, Phys. Fluids 11, 1596 (1999).

[29] J. A. Domaradzki and K.-C. Loh, Phys. Fluids 11, 2330 (1999).

[30] S. Stolz and N. A. Adams, Phys. Fluids 11, 1699 (1999).

[31] B. Vreman, B. Geurts, and H. Kuerten, Theor. Comput. Fluid Dyn. 8, 309 (1996).

[32] C. Härtel and L. Kleiser, J. Fluid Mech. 356, 327 (1998).

[33] M. Tsubokura, Phys. Fluids 13, 500 (2001).

[34] M. Ida and N. Taniguchi, in preparation.

[35] S. Grossmann, Rev. Mod. Phys. 72, 603 (2000).

[36] T. Elperin, N. Kleeorin, and I. Rogachevskii, Phys. Rev. E 68, 016311 (2003).

[37] P. Moeleker and A. Leonard, J. Comput. Phys. 167, 1 (2001).

[38] W. Yeo, Ph.D. thesis, Ohio State University, 1987 (unpublished). 